Doug Geisler, Eva K. Grebel, and Dante Minniti, eds.

\title{
The Top-Heavy Mass Function of the Young Super Star Cluster M82-F
}

\author{
Linda J. Smith \\ Department of Physics and Astronomy, University College London \\ Gower St., London WC1E 6BT, UK \\ John S. Gallagher III \\ Department of Astronomy, University of Wisconsin-Madison, 5534 \\ Sterling, 475 North Charter St., Madison WI 53706, USA
}

\begin{abstract}
We derive a dynamical mass of $1.2 \pm 0.1 \times 10^{6} \mathrm{M}_{\odot}$ for the young SSC M82-F and determine that the current $\left(L_{V} / M\right)_{\odot}$ is a factor of $\sim 5$ higher than that predicted by a standard Kroupa (2001) IMF. This suggests that M82-F has a deficit of low mass stars and will not survive to become an old globular cluster.
\end{abstract}

\section{Introduction and Observations}

M82 is one of the nearest examples of a spectacular starburst galaxy. Observations with $H S T$ reveal that hundreds of super star clusters (SSCs) reside in the starburst region (O'Connell et al. 1995; de Grijs, O'Connell, \& Gallagher 2001). SSCs are often associated with starbursts and it is suggested that they represent young globular clusters. One critical aspect is whether they have sufficient low mass stars to remain as bound systems over long time-scales.

We have obtained high dispersion $\left(8 \mathrm{~km} \mathrm{~s}^{-1}\right)$ echelle spectroscopy of the very luminous, young SSC ' $F$ ' in M82 with with the 4.2-m William Herschel Telescope (WHT), for the purpose of deriving its dynamical mass and assessing whether it will survive to become an old globular cluster. In a previous paper (Gallagher \& Smith 1999), we derived an age for M82-F of $60 \pm 20$ Myr by comparing its observed spectrum with theoretical model cluster spectra.

Aperture photometry was performed on M82-F using archival HST/WFPC2 images. We obtain $V=16.12 \pm 0.10$ and a half-light radius of $2.8 \pm 0.3 \mathrm{pc}$ for an adopted distance of $3.6 \mathrm{Mpc}$. We derive $E(B-V)=0.9 \pm 0.1$ towards M82$\mathrm{F}$ from comparison of the observed $(B-V)$ and $(V-I)$ colours with those predicted for a $60 \mathrm{Myr}$ old cluster from spectral synthesis models.

\section{Derived Parameters}

We have measured the line-of-sight velocity dispersion of M82-F by cross-correlating the red spectrum with the spectra of suitable template stars observed at the same time. We find that the closest spectral match is to an F8 II type star which is consistent with the age of the cluster. We obtain a velocity dispersion of 
$13.4 \pm 0.7 \mathrm{~km} \mathrm{~s}^{-1}$ and a dynamical mass of $1.2 \pm 0.1 \times 10^{6} \mathrm{M}_{\odot}$ by application of the virial theorem. The corresponding mass density within the half-mass radius is $6.4 \pm 0.9 \times 10^{3} \mathrm{M}_{\odot} \mathrm{pc}^{-3}$. We also obtain $M_{V}=-14.5 \pm 0.3$, and derive a visual luminosity-to-mass ratio $\left(L_{V} / M\right)_{\odot}=45 \pm 13$. M82-F clearly has the luminosity and mass to qualify as a young globular cluster.

We compare our derived parameters for M82-F with two other SSCs, NGC 1569A and NGC 1705-1 in Table 1. We have derived up-dated masses and visual luminosities for these clusters. For NGC 1705-1, we have re-measured its radius from archival WFPC2 images and obtain $r_{\mathrm{h}}=1.6 \pm 0.4 \mathrm{pc}$ for an adopted distance of 5.3 Mpc (Tosi et al. 2001, in prep.).

Table 1. Comparison of Properties of Super Star Clusters

\begin{tabular}{|c|c|c|c|}
\hline Parameter & NGC 1569A & NGC 1705-1 & M82-F \\
\hline Age (Myr) & $4-10^{a}$ & $10-20^{b}$ & $60 \pm 20$ \\
\hline Metallicity $\left(\mathrm{Z}_{\odot}\right)$ & $0.20^{c}$ & $0.45^{d}$ & $1.0^{e}$ \\
\hline $\mathrm{M}_{V}(\mathrm{mag})$ & $-14.0 \pm 0.4^{a}$ & $-13.8 \pm 0.4$ & $-14.5 \pm 0.3$ \\
\hline $\mathrm{r}_{h}(\mathrm{pc})$ & $2.3 \pm 0.3^{a}$ & $1.6 \pm 0.4$ & $2.8 \pm 0.3$ \\
\hline$\sigma\left(\mathrm{km} \mathrm{s}^{-1}\right)$ & $15.7 \pm 1.5^{f}$ & $11.4 \pm 1.5^{g}$ & $13.4 \pm 0.7$ \\
\hline Mass $\left(\times 10^{6} \mathrm{M}_{\odot}\right)$ & $1.3 \pm 0.2$ & $0.48 \pm 0.12$ & $1.2 \pm 0.1$ \\
\hline$\left(L_{V} / M\right)_{\odot}$ & $26 \pm 11$ & $60 \pm 24$ & $45 \pm 13$ \\
\hline
\end{tabular}

${ }^{a}$ Hunter et al. (2000); ${ }^{b}$ Heckmann \& Leitherer (1997); ${ }^{c}$ Kobulnicky \& Skillman (1997); ${ }^{d}$ Devost, Roy \& Drissen (1997); ${ }^{e}$ McLeod et al. (1993); ${ }^{f}$ Ho \& Filippenko (1996a); ${ }^{g}$ Ho \& Filippenko (1996b)

\section{Discussion and Conclusions}

To investigate the form of the present-day mass function (MF) for M82-F and the two other SSCs, we have calculated $\left(L_{V} / M\right)_{\odot}$ as a function of age for various IMF parameterizations with the Starburst99 code (Leitherer et al. 1999), allowing for mass loss from the cluster due to stellar winds and supernovae.

We adopt the parameterization of Kroupa (2001) to represent our IMF model: $\alpha=1.3$ for masses in the range $0.1 \leq M<0.5 \mathrm{M}_{\odot}$ and $\alpha=2.3$ for $M \geq 0.5 \mathrm{M}_{\odot}$ with an upper mass limit of $100 \mathrm{M}_{\odot}$, and lower mass limits $m_{1}$ in the range $0.1-3 \mathrm{M}_{\odot}$. These models are shown in Fig. 1. It is clear that there is a fundamental mismatch by a factor of $\sim 5$ between the derived $\left(L_{V} / M\right)_{\odot}$ for M82-F and that predicted by a standard Kroupa (2001) IMF with $m_{1}=0.1 \mathrm{M}_{\odot}$.

Sternberg (1998) finds that NGC 1569A has a steep MF with $\alpha \sim 2.5$, extending to $0.1 \mathrm{M}_{\odot}$. This is confirmed in Fig. 1 where we show $\left(L_{V} / M\right)_{\odot}$ for the Sternberg (1998) IMF parameters. For NGC 1705-1, Sternberg (1998) finds that the IMF is either flat or truncated. Our revised $\left(L_{V} / M\right)_{\odot}$ for this cluster is lower by a factor of 2 but it still lies well above the standard curve, indicating that NGC $1705-1$ probably has a truncated MF with $m_{l} \approx 1-3 \mathrm{M}_{\odot}$.

The high $\left(L_{V} / M\right)_{\odot}$ that we have derived for M82-F indicates that this SSC has an abnormal MF with a lower mass cut-off of 2-3 $\mathrm{M}_{\odot}$ to match the observations. This then implies a 'top-heavy' MF in the tightly gravitationally bound regions. The cluster is expected to dissolve within the next 1-2 Gyr. 


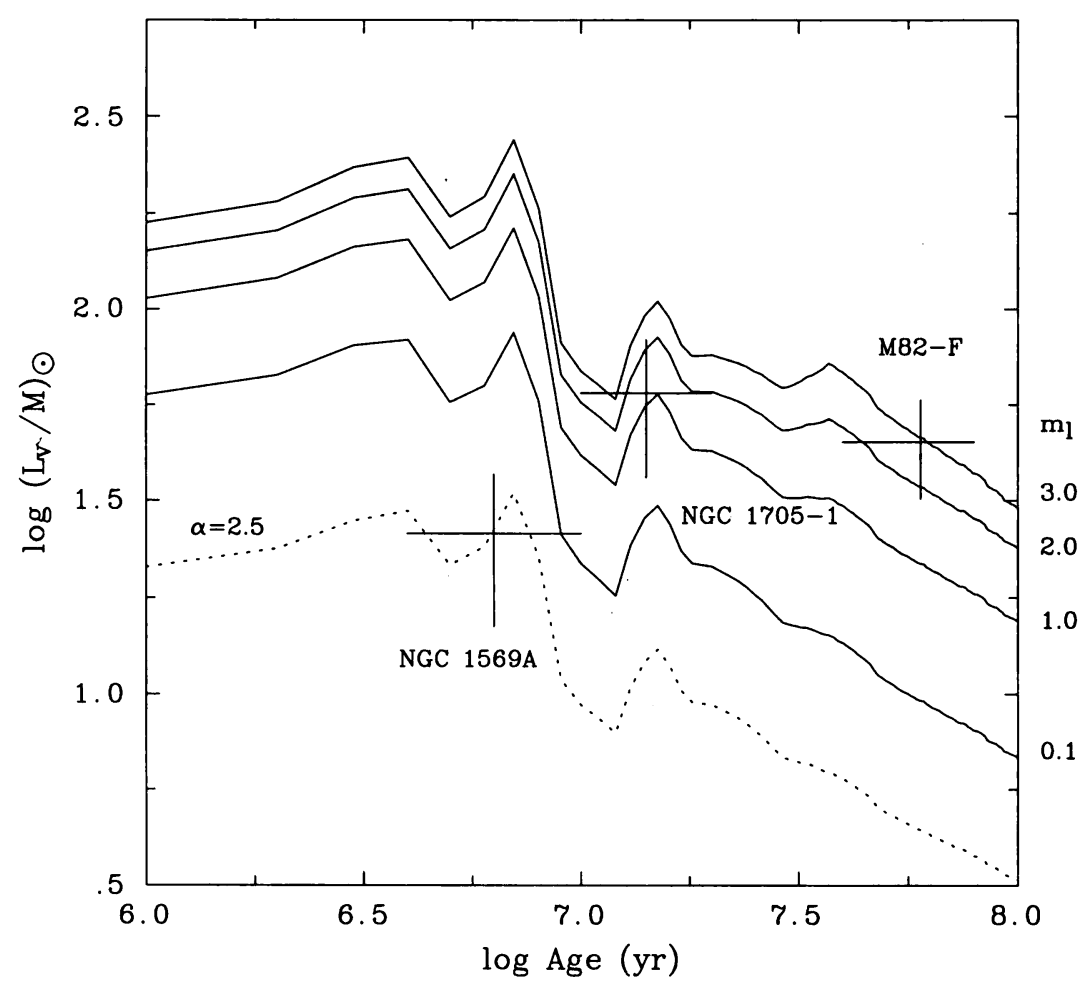

Figure 1. $\quad\left(L_{V} / M\right)_{\odot}$ plotted against age using the Starburst99 code. The solid curves are for a Kroupa (2001) IMF with $m_{\text {lower }}=0.1,1.0,2.0$ and $3.0 \mathrm{M}_{\odot}$ (see text for details). The derived $\left(L_{V} / M\right)_{\odot}$ and age values for M82-F, NGC 1569A and NGC 1705-1 are plotted. The dotted curve is for a slope of 2.5 and a lower mass cut-off of $0.1 \mathrm{M}_{\odot}$. 
The high mass and density of this cluster are not sufficient to assure its survival without sufficient mass in the form of long-lived, low mass stars.

\section{References}

Devost, D., Roy, J.-R., \& Drissen, L. 1997, ApJ, 482, 765

Gallagher, J.S., III, \& Smith, L.J. 1999, MNRAS, 304, 540

de Grijs, R., O'Connell, R.W., \& Gallagher, J.S. 2001, AJ, 121, 768

Heckman, T.M., \& Leitherer, C. 1997, AJ, 114, 69

Ho, L.C., \& Filippenko, A.V. 1996a, ApJ, 466, L83

Ho, L.C., \& Filippenko, A.V. 1996b, ApJ, 472, 600

Hunter, D.A., O’Connell, R.W., Gallagher, J.S., \& Smecker-Hane, Y.T. 2000, AJ, 120, 2383

Kobulnicky, H. A., \& Skillman, E. D. 1997, ApJ, 489, 636

Kroupa, P. 2001, MNRAS, 322, 231

Leitherer, C. et al. 1999, ApJS, 123, 3

McCleod, K.K., Rieke, G.H., Rieke, M.J., \& Kelly, D.M. 1993, ApJ, 412, 111

O'Connell, R.W., Gallagher, J.S., III, Hunter, D.A., \& Colley, W.N. 1995, ApJ, 446, L1

Sternberg, A. 1998, ApJ, 506, 721 